\title{
ECOG 2010 and beyond: taking childhood obesity off the menu
}

Childhood obesity is one of Europe's most serious public health challenges. Around $20 \%$ of European children are overweight and one-third of them obese. And as childhood obesity has the status of a disease, it is now classified as an epidemic. The prevalence of childhood overweight and obesity is already so high in Europe that obesity-related diseases and complications in later life, such as type 2 diabetes, CVD, cancers and psychosocial disorders, may very well lead to many European children living much shorter lives than their parents. This is a serious concern for the European Childhood Obesity Group (ECOG). ECOG brings together over 150 leading researchers, medical and public health professionals in the field of childhood obesity from across Europe. Our mission is to help the European community at large to understand fully the health, social, psychological and economic impacts of childhood obesity, and work together to take this growing problem off the menu in Europe.

In 2010 we have celebrated the 20th year anniversary of the ECOG and in November we hosted our largest congress to date, bringing together hundreds of childhood obesity experts and young scientists from across Europe to Brussels to discuss new developments, priorities and ways forward for the European Union (EU)'s new institutional framework. Our hope was to act as catalyst, bringing together many more stakeholders and contributing constructively to the work of the new European Parliament and new European Commission on this issue for the next 5 years. The year 2010 was also a key year for the European Commission's own agenda on obesity, with the review of the White Paper on Obesity planned for mid-2010. Indeed we invited the European Commission to think seriously about childhood obesity in the review. There are many more approaches needed and a great deal more that can and should be done.

\section{Childhood obesity in Europe: the drivers and main issues}

\section{Baby fat: obesity in preschool children}

'Baby fat', has long been the symbol of a thriving and healthy infant. This seemingly excess weight (which is actually quite normal) should quickly disappear if children are exclusively breast-fed for at least 6 months (the WHO recommendations), if appropriate weaning practices are used (including complementing the introduction of solid food with breast milk) and as children develop weight-bearing activities. The mounting evidence suggests that infancy is a critical period during which to prevent childhood obesity and its related consequences. A critical period is before the age of 5 years, when body fat normally should decline to a minimum before increasing again into adulthood. When this happens before 5 we face the 'early adiposity rebound' an early development of fat, clearly prognosing later in life obesity. The best thing we can do for children at this age is create ways of life that continue to make obesity unlikely. Measures for early intervention from parents and carers are needed, such as breast-feeding support and the introduction of lifestyles - eating habits, activity and good sleeping routines which, if continued are likely to prevent the development of obesity. Parents and carers also need to be supported, as such better education programmes to target these groups are needed, and health professionals in particular need to respect and follow the normal growth patterns for children by using appropriate growth standards. The ECOG officially endorsed the growth standards of the WHO.

\section{Enabling healthy lifestyles for children: a community approach}

Adult obesity is far more prevalent in socially disadvantaged groups; this is a common trend across Europe. Children are also more likely to be in a higher weight category than those from the most affluent families if they live in a socially disadvantaged area or an area of a lower socio-economic status. This does not necessarily stem from ignorance or choice: most families understand or at least have heard about the importance of a healthy lifestyle. Yet this problem is unfortunately linked to many others and poor nutrition is just the tip of the iceberg. Making what may seem like simple changes to daily habits (physical and nutritional) is simply too difficult given all the other difficulties many families have to confront.

This is just one example of a need for a community approach. As well as local programmes targeting specific groups, an overall strategy is needed to reduce the social gradients of health and to facilitate and promote lifestyle change, which is accessible to all groups, even disadvantaged people. People need to feel empowered to make change.

More research is needed into how to successfully reduce the social gradients of health and also into why disadvantaged or low-income groups are more at risk of being overweight. Socio-economic measures to facilitate acceptance of healthier lifestyles and to support communities to make positive changes need to be identified. Also more 
sound research is needed into what preventive or therapeutic measures work and why for overweight/obese children in these groups in particular, taking a global approach to a healthy lifestyle. Healthy lifestyles touch upon every facet of our daily lives and the lifestyle changes needed to prevent or manage childhood obesity (and adult obesity) have a great deal in common with the changes needed to tackle many of today's global problems - health and others. Measures to prevent CVD, some cancers, to promote mobility, sustainable living and even to reduce global warming all need to take healthy lifestyles into account. Currently, many of these problems tend to be considered separately and the result is a plethora of recommendations and programmes, all of which might be much more effective if brought together under one umbrella. Efforts to prevent childhood obesity should not be made in a silo. They should be part of the whole gamut of actions to reduce other 'chronic diseases' and to promote more sustainable living. There should be more open linkage between actions, reports, campaigns, resolutions and European-funded programmes on these issues so that Europeans can be given one holistic concept for a sustainable healthy way forward, rather than be bombarded and possibly disoriented by numerous and apparently different campaigns and programmes.

\section{Educating health workers}

Despite the publicity surrounding the childhood obesity epidemic, many health workers are poorly trained and/or badly equipped for managing childhood weight and obesity problems. They may have difficulty recognising the problem early on and they are often insufficiently trained to explain these issues to parents who often see this issue as a sensitive taboo. They may have inadequate time or resources to be able to advise families with overweight children and there may be no local facility to which they can refer children. There is a need for more practical training programmes for healthcare professionals at all levels - primary to tertiary - to recognise weight problems and obesity early on and to develop local programmes to manage overweight children.

There is also a need for more widespread facilities devoted to the management of overweight children in the community. A common educational approach for EU health-professionals is needed.

\section{From farm to fork and from fork to farm}

Health issues and healthy lifestyles should be actively included in the agriculture sector, in particular horticulturalists. The high cost of healthy food can make the socio-economic gap related to obesity development and other health issues much wider. It is important to have a wide and affordable choice of fresh fruit and vegetables available to all citizens, and not just those with more spending power. We propose a stronger alliance between the agricultural sector and health professionals in terms of research and communication to the general public on the importance of eating fruit and vegetables. We note the European Commission's efforts in this area, most recently DG Agriculture's Fruit in Schools Scheme, which is a very good start; however, we would ask the Commission to consider more coordinated incentives and better guidance for the agricultural sector for a longer-term impact in this area, both for health and the economic revenues of the sector.

\section{Advertising and marketing}

There is strong evidence to suggest that there is a direct relationship between advertising and higher rates of obesity in children. Much has been written on the subject and there could be room for further regulation. Industries need to be encouraged to work more on positive and more ethical programmes that put the health of consumers, and children in particular, first. Industry needs to think of its social as well as financial gains by, e.g., improving the quality of food for everybody and not only for wealthier social subgroups. They also need to improve food labelling by providing clearer and more consistent health claims on their products. We encourage joint-working between the food industry, consumer groups, the media and public health workers to develop positive and practical ideas for promoting sustainable healthy and enjoyable lifestyles for communities. By working together all parties can recognise their role and play to their strengths and conflict can be avoided. We invite the Commission to think seriously about this in their review of the White Paper on Obesity in 2010.

\section{Taking stock of existing material}

A plethora of projects and studies on overweight/obese children exists in the scientific and policy community in Europe. Yet almost all these studies suffer from limitations; the numbers of children studied may be relatively few, periods of follow-up are often short and the necessary follow-up years after interventions have finished is missing. There is need for an objective assessment of projects and studies in order to develop an objective evaluation of what works in practice. More and better coordination between projects is needed across all DG of the European Commission and International Organisations to avoid replication and to enable the outcomes of these projects to be useful for others.

\section{Conclusion}

ECOG is concerned for the healthy lives of children. We are also keen to ensure that Europe is doing its best to help reduce the rates of childhood obesity. This can be done in many different ways: existing and future laws, policies, programmes and campaigns. We invite the 
European Institutions to think seriously about the following issues in their review of the White Paper on Obesity in 2010:

- Obesity in infancy - By promoting measures for early intervention from parents and carers and providing better education programmes to target these groups.

- Research is needed into how to successfully reduce the social gradients of health and into socio-economic measures to facilitate acceptance of healthier lifestyles.

- Efforts to prevent childhood obesity should not be made in a silo. They should be part of the whole range of actions to reduce other 'chronic diseases' and to promote sustainable living.

- There is a need for more practical training programmes for health-care professionals at all levels - primary to tertiary - to recognize weight problems and obesity early on and to develop local programmes to manage and prevent overweight children.

- We propose a stronger alliance between the agricultural sector and health professionals in terms of research and communication to the general public on the importance of eating fruit and vegetables.

- Joint-working between the food industry, consumer groups, the media and public health workers is needed to develop positive and practical ideas for promoting

sustainable healthy and enjoyable lifestyles for communities.

- There is need for an objective assessment of projects and studies in order to develop objective evaluation of what works in practice.

ECOG's members are Europe's leading experts on the issue of childhood obesity. Between us we bring together both clinical and public health programmes, we have independence and authority and decades of experience in the field of childhood health and nutrition.

We can and want to become a constructive partner for the European Institutions on the issue of childhood obesity, sharing our work, providing a sound board and helping the Institutions themselves coordinate their efforts to combat this growing problem.

Healthy sustainable lifestyles are enjoyable and can contribute to happy, well balanced and more fulfilled individuals. We hope the information we provide in this paper gives you interesting (and healthy) food for thought. We look forward to working with you to take childhood obesity off the menu in Europe.

Walter Burniat, MD

20th ECOG Workshop

President
Margherita Caroli, MD PhD ECOG President

\title{
1st Workshop: A Common Approach and Tools for Paediatric Obesity and Treatment - Keynote Speaker Management of obesity in the very young
}

\author{
Marie Josée Mozin \\ Founder member and Honorary President of the European Club of Paediatric Dieticians
}

Overweight and obesity during infancy and early childhood need to be considered as potentially health threatening as well as a higher risk factor for the development of obesity in childhood and adolescence. There are different opinions among health professionals. Some propose to postpone nutritional interventions until 3 years of age whereas others recommend a controlled diet and the promotion of a healthy lifestyle as soon as weight excess appears. The following questions are major issues:

- Are overweight infants and toddlers at risk of developing immediate health problems?

- Do early interventions help to establish lifelong healthful behaviours and prevent obesity in later life?

- What are the nutritional risks related to dietary restrictions?
Morbidity is described in overweight children as well as in adults but less in infancy and in early childhood. Nevertheless, studies show more frequent developmental delays, mainly delayed gross motor skills, breathing difficulties like asthma, sleep apnoeas and other dyssomnias. It is commonly admitted that parents provide both genes and environment. The child's food choice is dependent on its parents own food preferences. Furthermore, eating behaviour is influenced by the environmental conditions during the meals. Familial tensions, sibling's attitude, noise, television viewing and organoleptic food characteristics may enhance or diminish the intakes and determine their specificities. Adults serve as model and may also influence food habits in terms of quality and quantity. Early eating 